\title{
DYNAMICAL BEHAVIOR OF THE STRONG DISPERSIVE NONLINEAR WAVE EQUATION*
}

\section{MEHMED KODZHA}

ABSTRACT: In this paper we consider the dynamical behavior of solutions near explicit self-similar solutions for a strong dispersive nonlinear wave equation. First we construct explicit self-similar solutions, then we investigate dynamical behavior of the solutions near to the self-similar solutions.

KEYWORDS: Strong dispersive nonlinear wave equation, Self-similar solutions, Dynamical behavior

2020 Math. Subject Classification: $35 C 06$

\section{Introduction}

In this paper we consider the following strong dispersive nonlinear wave equation

$$
u_{t}-\alpha^{2} u_{t x x}+2 k u_{x}+3 u u_{x}+\gamma\left(u-\alpha^{2} u_{x x}\right)_{x x x}=\alpha^{2}\left(2 u_{x} u_{x x}+u u_{x x x}\right),
$$

Equation (1.1) is a version of the following well-known generalization of the Dullin-Gottwald-Holm equation [1]

$$
u_{t}-\alpha^{2} u_{t x x}+2 \omega u_{x}+3 u u_{x}+\gamma u_{x x x}=\alpha^{2}\left(2 u_{x} u_{x x}+u u_{x x x}\right),
$$

Equation (1.2) is derived in [1] as a model for shallow water waves. The Cauchy problem for the equation Dullin-Gottwald-Holm in both periodic and non periodic case was studied in [4, 5, 6]. For (1.2) the problem of the asymptotic stability of self-similar solution was considered in [3]. The authors construct explicit self-similar solutions and consider

*This paper is (partially) supported by Scientific Research Grant RD-0842/2021 of Shumen University 
the dynamical behavior of the solutions near to the self-similar solutions. Moreover the asymptotic stability also was considered. Our aim in this paper to construct self-similar solutions of equation (1.1) and to consider dynamical behavior of the solutions arround of these solutions.

Paper is organizated as follows. In section 2 we construct the explicit form the self-similar solutions. In section 3 we investigate dynamical behavior of the solutions near to the self-similar solutions.

\section{The explicit self-similar solutions}

In this section, we construct self-similar solutions for equation

$$
u_{t}-\alpha^{2} u_{t x x}+2 k u_{x}+3 u u_{x}+\gamma\left(u-\alpha^{2} u_{x x}\right)_{x x x}=\alpha^{2}\left(2 u_{x} u_{x x}+u u_{x x x}\right),
$$

$(t, x) \in \mathbb{R}^{+} \times \mathbb{R}$.

Let the parameter $\mathrm{T}$ be a positive constant. We introduce the similarity coordinates

$$
\tau=-\log (T-t), \rho=\frac{x}{T-t}
$$

then we denote by

$$
u(t, x)=\phi\left(-\log (T-t), \frac{x}{T-t}\right),
$$

direct computation gives that

$$
\begin{aligned}
& u_{t}(t, x)=e^{\tau}\left(\phi_{\tau}+\rho \phi_{\rho}\right), u_{x}(t, x)=e^{\tau} \phi_{\rho}, \\
& u_{x x}(t, x)=e^{2 \tau} \phi_{\rho \rho}, u_{t x x}=e^{3 \tau}\left(\phi_{\tau \rho \rho}+2 \phi_{\rho \rho}+\rho \phi_{\rho \rho \rho}\right), \\
& u_{x x x}(t, x)=e^{3 \tau} \phi_{\rho \rho \rho}, u_{x x x x}(t, x)=e^{5 \tau} \phi_{\rho \rho \rho \rho \rho} .
\end{aligned}
$$

Thus Eq. (1.1) is transformed into an one dimensional quasilinear equation

$$
\begin{aligned}
& \phi_{\tau}+(\rho+2 k+3 \phi) \phi_{\rho}-\alpha^{2} e^{2 \tau}\left(\phi_{\tau \rho \rho}+2 \phi_{\rho \rho}+\rho \phi_{\rho \rho \rho}\right) \\
& +\gamma e^{2 \tau}\left(\phi-\alpha^{2} e^{2 \tau} \phi_{\rho \rho}\right)_{\rho \rho \rho}=\alpha^{2} e^{2 \tau}\left(2 \phi_{\rho} \phi_{\rho \rho}+\phi \phi_{\rho \rho \rho}\right) .
\end{aligned}
$$


The steady equation of quasilinear Eq. (2.2) is

$$
(\rho+2 k+3 \phi) \phi_{\rho}=0
$$

which is an ODE. Direct computation shows that it admits a non-trivial solution

$$
\phi(\rho)=-\frac{1}{3}(\rho+2 k)
$$

Consequently, the Eq. (1.1) admits an explicit self-similar solution

$$
u(t, x)=-\frac{1}{3}\left(\frac{x}{T-t}+2 k\right) .
$$

\section{Dynamical behavior}

Consider the perturbation of the form

$$
u(t, x)=v(t, x)+\bar{u}(t, x)
$$

where $\bar{u}(t, x)=-\frac{1}{3}\left(\frac{x}{T-t}+2 k\right)$ is the explicit self-similar solution given in (1.1).

We substitute (3.1) into (1.1), then a dissipative quasilinear equation with singular time coefficient is obtained as

$$
\begin{aligned}
& v_{t}-\alpha^{2} v_{t x x}-\gamma \alpha^{2} v_{x x x x x}+\left(\gamma+\frac{\alpha^{2}}{3}\left(\frac{x}{T-t}+2 k\right)\right) v_{x x x} \\
& +\frac{2 \alpha^{2}}{3(T-t)} v_{x x}-\frac{x}{T-t} v_{x}-\frac{1}{T-t} v \\
& =\alpha^{2}\left(2 v_{x} v_{x x}+v v_{x x x}\right)-3 v v_{x}, \quad \forall(t, x) \in(0, T) \times \mathbb{R},
\end{aligned}
$$

with the initial data $v(0, x)=v_{0}(x)=u_{0}(x)+\frac{1}{3}\left(\frac{x}{T}+2 k\right)$. 
In the similarity coordinates (2.1), Eq. (3.2) can be rewritten as follows

$$
\begin{aligned}
& v_{\tau}-\alpha^{2} e^{2 \tau} v_{\tau \rho \rho}-\frac{4 \alpha^{2}}{3} e^{2 \tau} v_{\rho \rho}+e^{2 \tau}\left(\gamma+\frac{2 \alpha^{2}}{3}(k-\rho)\right) v_{\rho \rho \rho} \\
& -\alpha^{2} \gamma e^{4 \tau} v_{\rho \rho \rho \rho \rho}-v+3 v v_{\rho}=\alpha^{2} e^{2 \tau}\left(2 v_{\rho} v_{\rho \rho}+v v_{\rho \rho \rho}\right) .
\end{aligned}
$$

We introduce the transformation $\bar{v}\left(\tau, \rho_{0}\right)=e^{-\tau} v(\tau, \rho)$, with $\rho_{0}:=e^{-\tau} \rho$, to reduce Eq. (3.3) into

$$
\begin{gathered}
\bar{v}_{\tau}-\alpha^{2} \bar{v}_{\tau \rho_{0} \rho_{0}}-\frac{\alpha^{2}}{3} \bar{v}_{\rho_{0} \rho_{0}}+e^{-\tau}\left(\gamma\left(\bar{v}-\alpha^{2} \bar{v}_{\rho_{0} \rho_{0}}\right)_{\rho_{0} \rho_{0} \rho_{0}}+\frac{2 \alpha^{2}}{3}(k-\rho) \bar{v}_{\rho_{0} \rho_{0} \rho_{0}}\right) \\
+\alpha^{2} \rho_{0} \bar{v}_{\rho_{0} \rho_{0} \rho_{0}}+\left(3 \bar{v}-\rho_{0}\right) \bar{v}_{\rho_{0}}=\alpha^{2}\left(2 \bar{v}_{\rho_{0}} \bar{v}_{\rho_{0} \rho_{0}}+\bar{v} \bar{v}_{\rho_{0} \rho_{0} \rho_{0}}\right)
\end{gathered}
$$

Note the operator $1-\alpha^{2} \partial_{\rho_{0} \rho_{0}}$ has a fundamental solution $p(x)=\frac{1}{2 \alpha} e^{-\left|\frac{\rho_{0}}{\alpha}\right|}$.

We can denote the operator $\left(1-\alpha^{2} \partial_{\rho_{0} \rho_{0}}\right)^{\frac{1}{2}}$ by $\Lambda$, then $\Lambda^{-2} \bar{v}=$ $p\left(\rho_{0}\right) \star \bar{v}$ for all $\bar{v} \in \mathbb{L}^{2}$.

Let $w\left(\tau, \rho_{0}\right)=\bar{v}\left(\tau, \rho_{0}\right)-\alpha^{2} \bar{v}_{\rho_{0} \rho_{0}}\left(\tau, \rho_{0}\right)$, then it holds $\bar{v}\left(\tau, \rho_{0}\right)=$ $p \star w$, where $\rho_{0} \in \mathbb{R}$ and $\star$ denotes the convolution. Furthemore, Eq. (3.3) can be rewritten as a dissipative non-local equation

$$
\begin{aligned}
& w_{\tau}+\frac{1}{3} w-e^{-\tau}\left(\frac{2 k+e^{\tau} \rho_{0}}{3}\right) w_{\rho_{0}}+\gamma e^{-\tau} w_{\rho_{0} \rho_{0} \rho_{0}}-\frac{1}{3}(p \star w) \\
& +e^{-\tau}\left(\frac{2\left(k-e^{\tau} \rho_{0}\right)}{3}\right)(p \star w) \rho_{0}+3(p \star w)(p \star w) \rho_{0} \\
& =2(p \star w) \rho_{0}(p \star w-w)+(p \star w)\left((p \star w) \rho_{0}-w_{\rho_{0}}\right),
\end{aligned}
$$

with the initial data

$$
\begin{aligned}
w\left(0, \rho_{0}\right):=w_{0}\left(\rho_{0}\right) & =v_{0}\left(\rho_{0}\right)-\alpha^{2} v_{\rho_{0} \rho_{0}}\left(0, \rho_{0}\right) \\
& =u_{0}(x)-\alpha^{2} u_{0}^{\prime}(x)+\frac{1}{3}\left(\frac{x}{T}+2 k\right) \\
& -6-
\end{aligned}
$$


and the boundary condition

$$
\lim _{\left|\rho_{0}\right| \rightarrow+\infty} w\left(\tau, \rho_{0}\right)=0, \quad \lim _{\left|\rho_{0}\right| \rightarrow+\infty} w_{\rho_{0}}\left(\tau, \rho_{0}\right)=0
$$

Here we use $(p \star w) \rho_{0} \rho_{0}=\alpha^{-2}(p \star w-w)$.

The term $\left(1-\frac{a}{m}\right) w$ is a dissipative term in Eq. (3.4). This term can make us to get a good priori estimate on the solution for Eq. (3.4). We recall a commutator estimate established in [2].

Lemma 3.1. [2] Let $s>0$. Then it holds

$$
\left\|\left[\Lambda^{s}, u\right] v\right\|_{\mathbb{L}^{2}} \leq C\left(\left\|\partial_{x} u\right\|_{\mathbb{L}^{\infty}}\left\|\Lambda^{s-1} v\right\|_{\mathbb{L}^{2}}+\left\|\Lambda^{s} u\right\|_{\mathbb{L}^{2}}\|v\|_{\mathbb{L}^{\infty}}\right),
$$

where positive constant $C$ depending on $s$.

We now derive a priori estimate of the solution for Eq. (3.4). Let $s>0$. Applying $\Lambda^{s}$ to both sides of (3.4), it holds

$$
\begin{aligned}
& \left(\Lambda^{s} w\right)_{\tau}+\frac{1}{3} \Lambda^{s} w-e^{-\tau} \Lambda^{s}\left[\frac{2 k+e^{\tau} \rho_{0}}{3} w \rho_{0}\right]+\gamma e^{-\tau} \Lambda^{s} w \rho_{0} \rho_{0} \rho_{0} \\
& -\frac{1}{3} \Lambda^{s}(p \star w)+e^{-\tau} \Lambda^{s}\left[\frac{2\left(k-e^{\tau} \rho_{0}\right)}{3}(p \star w) \rho_{0}\right]+3 \Lambda^{s}(p \star w)(p \star w) \rho_{0} \\
& =2 \Lambda^{s}\left[(p \star w) \rho_{0}(p \star w-w)\right]+\Lambda^{s}\left[(p \star w)\left((p \star w) \rho_{0}-w \rho_{0}\right)\right] .
\end{aligned}
$$

Lemma 3.2. Let $s>4$. Then any solution $w$ of Eq. (3.4) satisfies

$$
\|w\|_{\mathbb{H}^{s}(\mathbb{R})} \leq C e^{-\tau}\left\|w_{0}\right\|_{\mathbb{H}^{s}(\mathbb{R})},
$$

where $C$ is a positive constant, depending on $s$. 
Proof. Taking the $\mathbb{L}^{2}$-inner product with equation (3.8) by $\Lambda^{s} w$, we get

$$
\begin{aligned}
& \frac{1}{2} \frac{d}{d \tau}\|w\|_{\mathbb{H}^{s}}^{2}+\frac{1}{3}\|w\|_{\mathbb{H}^{s}}^{2}-e^{-\tau} \int_{\mathbb{R}} \Lambda^{s} w \Lambda^{s}\left[\frac{2 k+e^{\tau} \rho_{0}}{3} w_{\rho_{0}}\right] d \rho_{0} \\
& +\gamma e^{-\tau} \int_{\mathbb{R}} \Lambda^{s} w \Lambda^{s}\left[w_{\rho_{0} \rho_{0} \rho_{0}}\right] d \rho_{0}-\frac{1}{3} \int_{\mathbb{R}} \Lambda^{s} w \Lambda^{s}(p \star w) d \rho_{0} \\
& +e^{-\tau} \int_{\mathbb{R}} \Lambda^{s} w \Lambda^{s}\left[\frac{2\left(k-e^{\tau} \rho_{0}\right)}{3}(p \star w) \rho_{0}\right] d \rho_{0} \\
& +3 \int_{\mathbb{R}} \Lambda^{s} w \Lambda^{s}\left((p \star w)(p \star w) \rho_{0}\right) d \rho_{0} \\
& =2 \int_{\mathbb{R}} \Lambda^{s} w \Lambda^{s}\left[(p \star w) \rho_{0}(p \star w-w)\right] d \rho_{0} \\
& +\int_{\mathbb{R}} \Lambda^{s} w \Lambda^{s}\left[(p \star w)\left((p \star w) \rho_{0}-w \rho_{0}\right)\right] d \rho_{0} .
\end{aligned}
$$

Next we estimate each of terms in (3.9) . On the hand, we use integration by parts to derive

$$
\begin{aligned}
& \int_{\mathbb{R}} \Lambda^{s} w \Lambda^{s}\left[\frac{2 k+e^{\tau} \rho_{0}}{3} w_{\rho_{0}}\right] d \rho_{0}=\int_{\mathbb{R}}\left[\frac{2 k+e^{\tau} \rho_{0}}{3} w_{\rho_{0}}\right] \Lambda^{2 s} w d \rho_{0} \\
& =-\frac{1}{3} e^{\tau} \int_{\mathbb{R}} \Lambda^{s} w \Lambda^{s} w d \rho_{0}-\frac{1}{2} \int_{\mathbb{R}} \frac{2 k+e^{\tau} \rho_{0}}{3}\left(\Lambda^{s} w\right)_{\rho_{0}}^{2} d \rho_{0} \\
& =-\frac{1}{6} e^{\tau}\|w\|_{\mathbb{H}^{s}}^{2},
\end{aligned}
$$

and

$$
\int_{\mathbb{R}} \Lambda^{s} w \Lambda^{s}\left[w_{\rho_{0} \rho_{0} \rho_{0}}\right] d \rho_{0}=-\frac{1}{2} \int_{\mathbb{R}}\left(\Lambda^{s} w_{\rho_{0}}\right)_{\rho_{0}}^{2} d \rho_{0}=0,
$$

and

$$
\frac{1}{3} \int_{\mathbb{R}} \Lambda^{s} w \Lambda^{s}(p \star w) d \rho_{0}=\frac{1}{3}\|w\|_{\mathbb{H}^{s-1}}^{2},
$$


and

$$
\int_{\mathbb{R}} \Lambda^{s} w \Lambda^{s}\left[\frac{2\left(k-e^{\tau} \rho_{0}\right)}{3}(p \star w) \rho_{0}\right] d \rho_{0}
$$

$$
\begin{aligned}
& =\frac{2}{3} e^{\tau} \int_{\mathbb{R}} \Lambda^{s-1} w \Lambda^{s-1} w d \rho_{0}+\frac{1}{2} \int_{\mathbb{R}} \frac{2\left(k-e^{\tau} \rho_{0}\right)}{3}\left(\Lambda^{s-1} w\right)_{\rho_{0}}^{2} d \rho_{0} \\
& =e^{\tau}\|w\|_{\mathbb{H}^{s-1}}^{2}
\end{aligned}
$$

and

$$
3 \int_{\mathbb{R}} \Lambda^{s} w \Lambda^{s}\left((p \star w)(p \star w) \rho_{0}\right) d \rho_{0}=-\frac{3}{2} \int_{\mathbb{R}} w_{\rho_{0}}\left(\Lambda^{s-1} w\right)^{2} d \rho_{0}
$$

$$
\leq \frac{3}{2}\left\|w_{\rho_{0}}\right\|{\mathbb{\mathbb { L } ^ { \infty }}}\|w\|_{\mathbb{H}^{s-1}}^{2} \leq \frac{3}{2}\|w\|_{\mathbb{H}^{s-1}}^{3} .
$$

On the other hand, by (3.7), Holder inequality and $\mathbb{H}^{s-1} \subset \mathbb{L}^{\infty}$ with $s>4$, we derive

$$
\begin{aligned}
& 2\left|\int_{\mathbb{R}} \Lambda^{s} w \Lambda^{s}\left((p \star w) \rho_{0}(p \star w-w)\right) d \rho_{0}\right| \\
& =2\left|\int_{\mathbb{R}}\left[\Lambda^{s},(p \star w-w)\right](p \star w) \rho_{0} \Lambda^{s} w d \rho_{0}\right| \\
& +2\left|\int_{\mathbb{R}}(p \star w-w) \Lambda^{s}(p \star w) \rho_{0} \Lambda^{s} w d \rho_{0}\right|
\end{aligned}
$$

$$
\begin{aligned}
& \leq C\left(\left\|(p \star w-w) \rho_{0}\right\|_{\mathbb{L}^{\infty}}\left\|\Lambda^{s-1}(p \star w) \rho_{0}\right\|_{\mathbb{L}^{2}}\right. \\
& \left.+\left\|\Lambda^{s}(p \star w-w)\right\|_{\mathbb{L}^{2}}\left\|(p \star w) \rho_{\rho_{0}}\right\|_{\mathbb{L}^{\infty}}\right)\|w\|_{\mathbb{H}^{s}} \\
& +2\left(\|p \star w-w\|_{\mathbb{L}^{\infty}}+\left\|(p \star w-w) \rho_{\rho_{0}}\right\| \|_{\mathbb{L}^{\infty}}\right)\|w\|_{\mathbb{H}^{2}}^{2} \\
& \leq C\|w\|_{\mathbb{H}^{s}}^{3}
\end{aligned}
$$


and

$$
\begin{aligned}
& \left|\int_{\mathbb{R}} \Lambda^{s} w \Lambda^{s}\left((p \star w)\left((p \star w) \rho_{0}-w_{\rho_{0}}\right)\right) d \rho_{0}\right| \\
& =\left|\int_{\mathbb{R}}\left[\Lambda^{s},(p \star w)\right]\left((p \star w) \rho_{0}-w_{\rho_{0}}\right) \Lambda^{s} w d \rho_{0}\right| \\
& +\left|\int_{\mathbb{R}}(p \star w) \Lambda^{s}\left((p \star w) \rho_{0}-w \rho_{0}\right) \Lambda^{s} w d \rho_{0}\right| \\
& \leq C\left(\left\|(p \star w) \rho_{0}\right\|_{\mathbb{L}^{\infty}}\left\|\Lambda^{s-1}\left((p \star w) \rho_{0}-w_{\rho_{0}}\right)\right\|_{\mathbb{L}^{2}}\right. \\
& \left.+\left\|\Lambda^{s}(p \star w)\right\|_{\mathbb{L}^{2}}\left\|(p \star w) \rho_{0}-w_{\rho_{0}}\right\|_{\mathbb{L}^{\infty}}\right)\|w\|_{\mathbb{H}^{s}} \\
& +2\|p \star w\|_{\mathbb{L}^{\infty}}\|w\|_{\mathbb{H}^{2}}^{2} \\
& \leq C\|w\|_{\mathbb{H}^{s}}^{3},
\end{aligned}
$$

where $C$ is a positive constant, depending on $s$.

Thus using (3.11)-(3.16), it follows from (3.9) that

$$
\frac{d}{d \tau}\|w\|_{\mathbb{H}^{s}}^{2}+\|w\|_{\mathbb{H}^{s}}^{2} \leq \frac{d}{d \tau}\|w\|_{\mathbb{H}^{s}}^{2}+\|w\|_{\mathbb{H}^{s}}^{2}+\frac{4}{3}\|w\|_{\mathbb{H}^{s-1}}^{2} \leq C\|w\|_{\mathbb{H}^{s}}^{3},
$$

which is a Bernoulli-type differential inequality, it is equivalent to

$$
-\frac{d}{d \tau}\|w\|_{\mathbb{H}^{s}}^{-1}+\|w\|_{\mathbb{H}^{s}}^{-1} \leq C
$$

which given that

$$
\|w\|_{\mathbb{H}^{s}} \leq C e^{-\tau}\left\|w_{0}\right\|_{\mathbb{H}^{s}}
$$

\section{REFERENCES:}

[1] Dullin H, Gottwald G, Holm D. An integrable shallow water equation with linear and nonlinear dispersion. Phy Rev Lett 2001; 87:194501. 4 
[2] Kato T. Quasi-linear equations of evolution, with applications to partial differential equations, in: Spectral theory and differential equations (proc. sympos.), Dundee, 1974. In: Lecture notes in Math., vol. 48. Berlin: Springer; 1975. p. 25-70. Dedicated to Konrad Jorgens

[3] Li H, Yan W. Asyptotic stability and instability of explicit self-similar waves for a class of nonlinear shallow water equations. Commun Nonlinear Sci Numer Simulat 79 (2019), 104928

[4] Liu Y. Global existence and blowup solutions for a nonlinear shallow water equation. Math Ann 2006; 335:717-35

[5] Tian LX, Gui GL, Liu Y. On the well-posedness problem and the scattering problem for the Dullin-Gottwald-Holm equation. Commun Math Phys 2005; 257:667-70

[6] Zhou Y. Blow-up of solutions to the DGH equation. J Functional Anal 2007; 250:227-48

\section{Mehmed Kodzha}

Konstantin Preslavsky University of Shumen

9712 Shumen, Bulgaria

e-mail: m.kodzhadshu.bg 